\title{
DESIGN OF EFFECTIVE FEATURE EXTRACTION IN UNDERWATER IMAGES USING DTCWT
}

\author{
Lini Lal1 ${ }^{1}$, Basavanagouda $\mathbf{G}^{2}$ \\ ${ }^{l}$ PG Student (VLSI Design and Embedded System), ECE, T. John Institute of Technology, Karnataka, India \\ ${ }^{2}$ Assistant Professor ECE, T. John Institute of Technology, Karnataka, India
}

\begin{abstract}
A stereo vision based strategy is utilized as a part of request to explore the unmanned submerged vehicle to find itself with the guide of natural reference data. Submerged picture experience the ill effects of non uniform brightening, obscuring and low differentiation and decreased hues. Picture upgrade must be done before doing further handling. The movement and pivot invariance properties of the DTCWT can be utilized to register exact and effective appraisals of the geometrical structure in pictures. By utilizing the edges recognized utilizing DTCWT calculation, the objects submerged can be identified and the UUVs can be explored in like manner.
\end{abstract}

Keywords: Underwater Image, Feature Extraction, DTCWT, Edge Detection

\section{INTRODUCTION}

In the course of the most recent couple of decades underwater imaging is a standout amongst the most critical exploratory fields of examination. Underwater vision gives a noteworthy and key part in a significant number of the naval force applications which incorporates mine location, jumper perceivability, pursuit and salvage, review of boat wrecks, pipelines, telecom links and coral reefs. Submerged environment is a truly complex situation for both the certain constrained availability and the trouble to recover great quality information. As a rule, laser frameworks and acoustic frameworks which are expensive contrasted with an optical imaging framework are utilized for these sorts of submerged applications. Be that as it may, when obvious light based imaging framework is utilized for submerged perception the expense and additionally multifaceted nature is diminished.

Unmanned underwater vehicles (UUVs) are utilized for military and in addition regular citizen applications. A stereo vision based technique is utilized as a part of request to explore the vehicle to find itself with the guide of natural reference data. Movement of the vehicle can be assessed utilizing the assistance of area data and in view of that the vehicle can be explored in a more secure and additionally proficient way. The cameras catch the symbolism of the surroundings containing the elements and these components go about as signals for route from one point to the next with an underlying referred to position of the vehicle as the reference. The area of the vehicle is registered utilizing the relative area of the indistinguishable components in progressive edges. In this way with camera as the main sensor, it is conceivable to assess the vehicle's position and introduction even without data from different sensors, and subsequently, this sort of route is called highlight based route. To finish this, enrollment of picture casings is completed which includes identification, extraction, and coordinating of elements between progressive edges.

Highlight recognition, depiction and coordinating of steady and discriminative components are major issues in PC vision. The component locators and descriptors assume a key part in numerous vision applications, for example, gauge coordinating, picture sewing, picture enrollment, picture recovery, robot restriction, video information mining, building scenes, surface acknowledgment, object identification and item acknowledgment. The noteworthy purpose of highlight point recognition is security and peculiarity that is, the assessed highlight point in the progressive pictures ought to be steady concerning the geometric and photometric varieties and it ought to be unmistakable.

The movement and pivot invariance properties of the DTCWT can be utilized to register exact and productive appraisals of the geometrical structure in pictures, to be specific the quality, introduction, and counterbalance of picture edges, edges, and different singularities. DTCWT likewise gives safety towards noise and permits huge edge discovery in a very noisy picture.

\subsection{Statement of The Problem}

Underwater imaging has been very much helpful in various military as well as civilian applications. Underwater images suffer from non uniform illumination, blurring and low contrast and diminished colors because light is attenuated as it travels in the water. So a novel algorithm is needed for object detection and recognition underwater. Significant objects should be detected and displayed accordingly.

\subsection{Scope of the Study}

In this paper an effective algorithm is developed to extract features from underwater images which can be very useful 
underwater autonomous navigation. The underwater vehicles with stereo based vision can navigate according to the objects detected by avoiding the obstacles in its path. Whenever an obstacle is detected, the vehicle can deviate its path and avoid colliding. The project also includes the use of DTCWT algorithm for edge detection which will result in improved noise performance.

\section{BLOCK DIAGRAM}

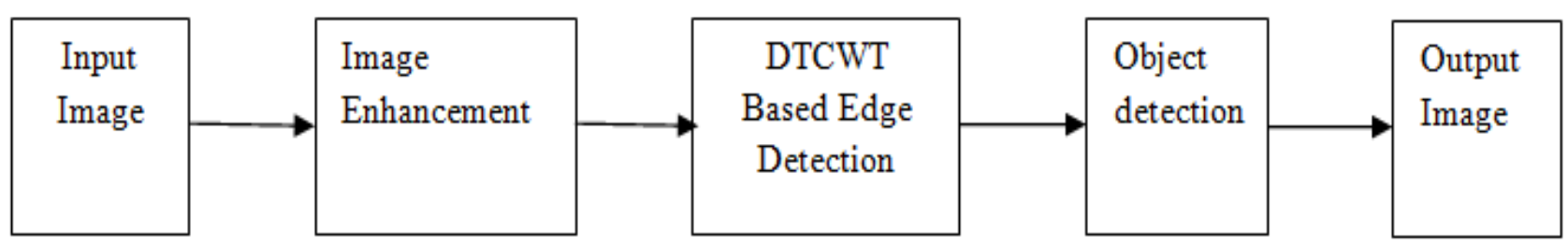

Fig-1: Block diagram

In feature extraction step at first the pre-processed and enhanced image is taken and edges are detected using DTCWT coefficients. The edge detected image is then thresholded to avoid low intensity pixels. Then the boundary
Underwater images are, are, in any case, experiencing picture quality corruption because of to the absorption and scattering of light. A few strategies have been proposed to improve the nature of symbolism recorded submerged. Such upgrades is an essentials step if more propelled utilizations of PC vision and photogrammetry, for example, highlight extraction, are to be fruitful in sea-going applications. Shading revision and complexity improvement will be done in picture upgrade strategy. The handled picture will be given for highlight extraction in which DTCWT calculation will be utilized.

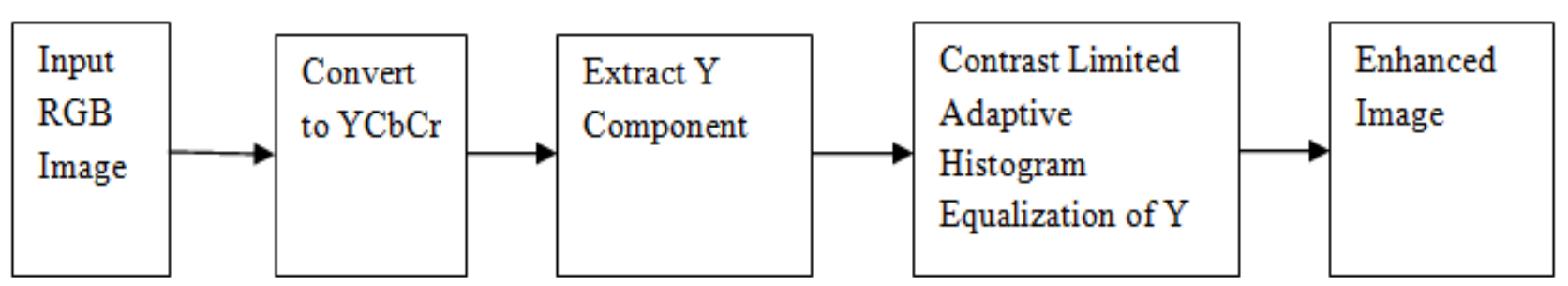

Fig -2: Image Enhancement block diagram

Underwater pictures regularly seem green-blue on the grounds that these shading parts are last consumed. The marvel of shading assimilation causes the caught submerged pictures to have low shading and difference execution. Besides, the essential data from the picture is likewise misfortune. To restore the complexity, shading, and misfortune data from the pictures, the utilization of PC vision and picture preparing are increasing essential. Contrast upgrade method is generally utilized for submerged picture preparing to enhance the differentiation execution. The improvement of the difference upgrade strategy for submerged picture has pulled in impressive consideration as of late. Scientists are enhancing the picture complexity to extricate whatever number data as would be prudent by applying different calculations. Various works have likewise been distributed based upon the models of subjective human shading vision that has the largest element scope of shading and complexity.

By converting the RGB image to $\mathrm{YCbCr}$ image more easily we can implement the contrast stretching. Contrast stretching can only be done to intensity images. If we are converting the image to $\mathrm{YCbCr}$, it is easy to convert it back to RGB and also the values can be used for further calculations. of the object found is extracted using some suitable algorithm and it is represented back in the enhanced image.

\subsection{Image Enhancement}

Contrast stretching uses the linear scaling function for the pixel values. It is used to enhance the contrast of the image. It is done by stretching the range of colour values to make use of all possible values. Each pixel is scaled using the function

$$
P_{0}=\left(P_{i}-c\right) \times(b-c) /(d-c)+a
$$

Where $\mathrm{P}_{\mathrm{o}}$ is the pixel value normalized;

$P_{i}$ is the pixel value considered;

$\mathrm{a}$ and $\mathrm{b}$ is the minimum and maximum desired range

$\mathrm{c}$ and $\mathrm{d}$ are the lowest and highest value of the current image.

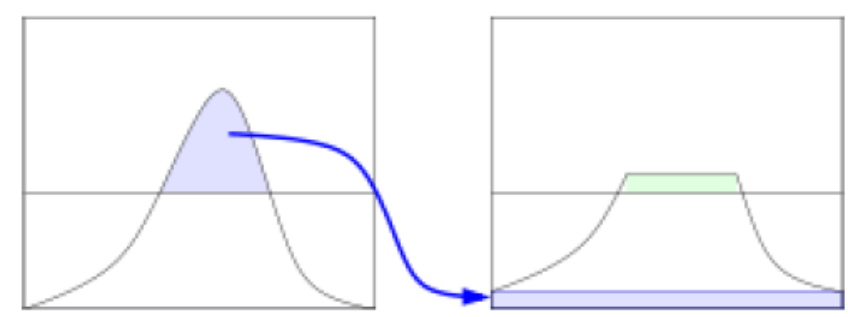

Fig-3: CLAHE contrast Stretching 


\subsection{DTCWT based Edge Detection}

\begin{tabular}{|c|c|c|c|c|}
\hline $\begin{array}{l}\text { Enhanced } \\
\text { Image }\end{array}$ & $\begin{array}{l}\text { Take } 5 \text { level } \\
\text { DTCWT of Y } \\
\mathrm{Cb} \& \mathrm{Cr} \\
\text { separately }\end{array}$ & $\begin{array}{l}\text { Take only } \\
\text { high pass } \\
\text { sub band }\end{array}$ & $\begin{array}{l}\text { Take } 5 \text { level } \\
\text { inverse DTCWT } \\
\text { of } \mathrm{Y} \mathrm{Cb} \& \mathrm{Cr} \\
\text { separately }\end{array}$ & $\begin{array}{l}\text { Threshold the } \\
\text { images and } \\
\text { combine } \mathrm{Y} \\
\mathrm{CbCr} \text { edges }\end{array}$ \\
\hline
\end{tabular}

Fig-4: DTCWT based edge Detection block diagram

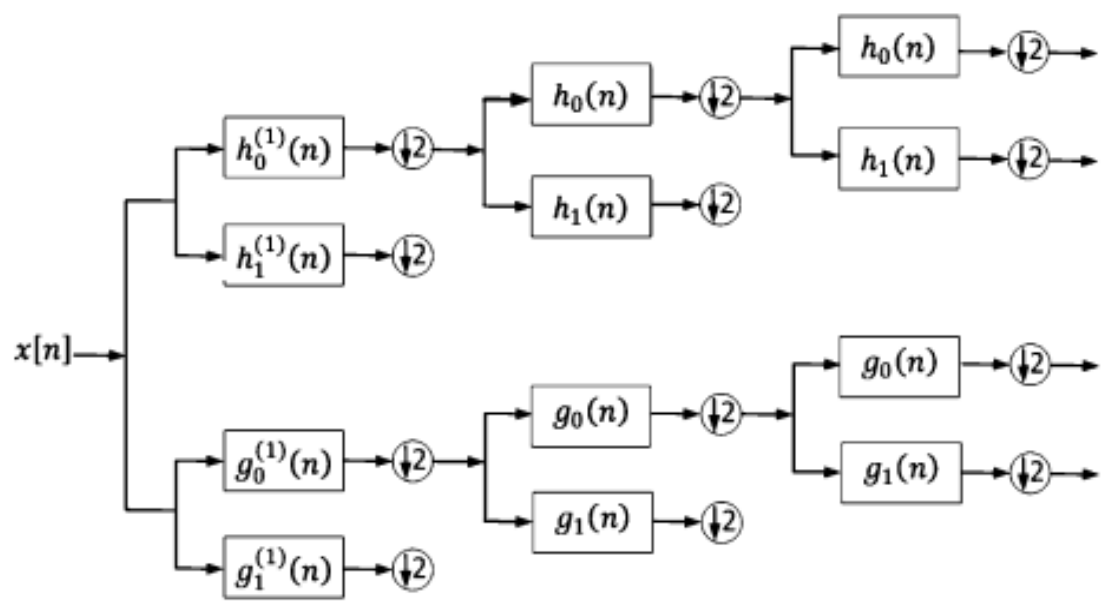

Fig-5: Structure of the analysis part of the DTCWT for 3 levels

For 2-D, picture force is regularly relative to scene brilliance, physical edges (comparing to the huge varieties in reflectance, light, introduction and profundity of scene surfaces) are spoken to in the picture by changes in the power work. Because of abundance of data connected with edges, edge identification is critical undertakings for some applications identified with PC vision and example acknowledgment. Edges are the high frequency components of an image.

DTCWT uses analytic filters to perform wavelet analysis. DTCWT uses two real DWT trees to implement its real tree (tree a) and imaginary (tree b) parts. DT-CWT decomposes a signal in terms of a complex shifted and dilated mother wavelet $\psi(\mathrm{x})$ and scaling function $\varphi(\mathrm{x})$. The real $(\mathrm{r})$ and imaginary (i) parts of the wavelet and scaling functions for one dimensional case can be described as

$$
\begin{aligned}
& \psi_{r}(t)=\sqrt{2} \sum_{n} H_{a}(n) \varphi_{r}(2 t-n) \\
& \psi_{i}(t)=\sqrt{2} \sum_{n} H_{b}(n) \varphi_{i}(2 t-n) \\
& \varphi_{r}(t)=\sqrt{2} \sum_{n} L_{a}(n) \psi(2 t-n) \\
& \varphi_{i}(t)=\sqrt{2} \sum_{n} L_{b}(n) \psi_{i}(2 t-n)
\end{aligned}
$$

Where $\mathrm{L}$ is the low pass filter and $\mathrm{H}$ is the high pass filter. Since as shown in fig 4 , the high frequency components are extracted in DTCWT, the edge detection can be achieved by taking only the high frequency sub band of the DTCWT algorithm.

\section{RESULTS}

After designing the whole system in MATLAB some promising results were obtained.
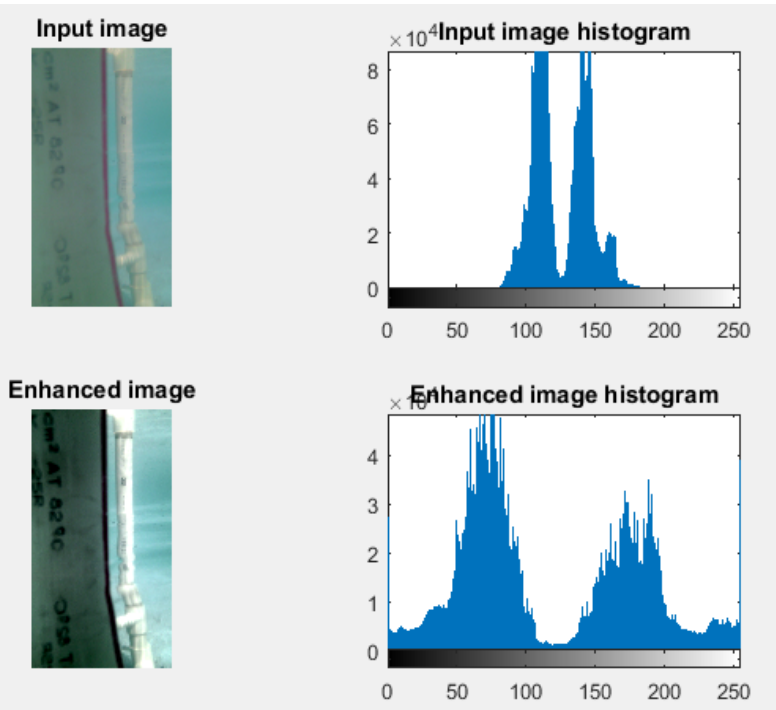

Fig-6: Image Enhancement block result 

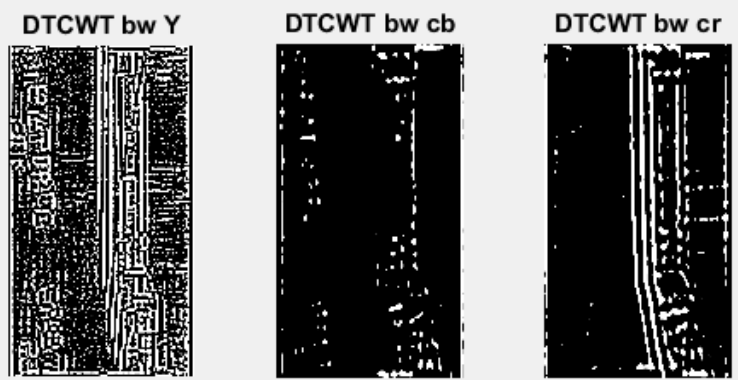

Fig-7: Edge Detection using DTCWT result

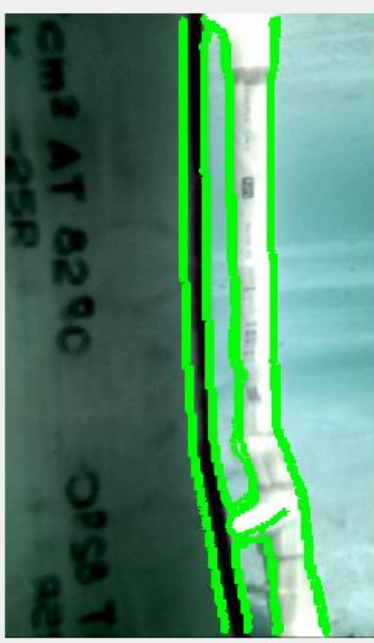

Fig -7: Object Detection

\section{CONCLUSION}

DTCWT algorithm is very robust in edge detection in the presence of noise in case of underwater images. Due to various underwater conditions some degradation will be there for images taken under water which can be rectified using CLAHE technique for underwater images. After that efficient feature extraction can be done using DTCWT

\section{ACKNOWLEDGEMENT}

I would like to take this opportunity to express our deepest gratitude and appreciation to all those who have helped me directly or indirectly towards the successful completion of this paper.

\section{REFERENCES}

[1]. Shubha Bhat, Ramesh Babu D.R., Krishnan Rangarajan, and Ramakrishna K.A "An Algorithm to Estimate Scale Weights of Complex Wavelets for Effective Feature Extraction in Aerial Images" Defence Science Journal, Vol. 64, No. 6, November 2014, pp. 549-556

[2]. C. J. Prabhakar, P. U. Praveen Kumar "Color Invariant Feature Detection and Matching in Underwater Stereo Images" Proceedings of the Fourth International Conference on Signal and Image Processing 2012
(ICSIP 2012) Volume 221 of the series Lecture Notes in Electrical Engineering pp 609-619 Date: 11 January 2013

[3]. Gorkem Serbes, Betul Erdogdu Sakar, Halil Ozcan Gulcur, Nizamettin Aydin, "An emboli detection system based on Dual Tree Complex Wavelet Transform and ensemble learning", Applied Soft Computing, Volume 37, December 2015, Pages 87-94.

[4]. W. Selesnick, R. G. Baraniuk and N. G. Kingsbury, "The Dual-Tree Complex Wavelet Transform."IEEE Signal Processing Magazine, vol. 22, no. 6, 2005.

\section{BIOGRAPHIES}

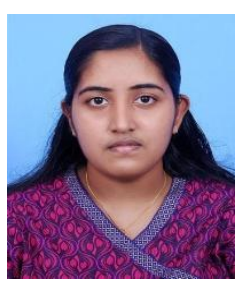

Mrs. Lini Lal completed her B.E. in ECE in the year 2011 and presently pursuing $M$. Tech in VLSI design and embedded systems. The area of interest is image processing and Verilog implementation.

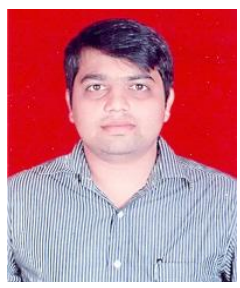

Mr. Basavanagouda G Asst. professor, Dept. of ECE, TJIT, Bengaluru-83. Has the experience in ASIC front end verification. Pursuing the $\mathrm{PhD}$ in the field of SOC implementation of VHT wireless modem. 